\title{
Zebrafish come modello per lo studio di malattie della tiroide
}

\author{
Federica Marelli ${ }^{1,2} \cdot$ Luca Persani $^{1,2}$
}

Accettato: 21 luglio 2020 / Pubblicato online: 24 febbraio 2021

(c) The Author(s) 2021

Sommario Le tecniche di sequenziamento di nuova generazione hanno rivoluzionato l'identificazione dei genimalattia, accelerando la scoperta di nuove mutazioni e nuovi geni candidati per le malattie della tiroide. Per far fronte a questo flusso di nuove informazioni genetiche è importante disporre di modelli animali adeguati per studiare i meccanismi che regolano lo sviluppo della tiroide, la biodisponibilità e l'azione degli ormoni tiroidei. Zebrafish (Danio rerio), con il suo rapido sviluppo embrionale esterno, è stato ampiamente utilizzato in biologia dello sviluppo. Ad oggi, quasi tutti i componenti dell'asse tiroideo zebrafish sono stati caratterizzati e sono strutturalmente e funzionalmente paragonabili a quelli dei vertebrati superiori. La disponibilità di linee transgeniche di zebrafish fluorescenti consente l'analisi in tempo reale dell' organogenesi tiroidea e delle sue alterazioni. Il knockdown transitorio ottenuto con l'uso del morfolino permette di silenziare l'espressione di un gene di interesse e ottenere prontamente informazioni sul suo contributo durante lo sviluppo dell'asse tiroideo in zebrafish. Gli strumenti recentemente disponibili per il knockout genico stabile (es. CRISPR/Cas9) hanno ulteriormente aumentato il valore di zebrafish nello studio della patologia tiroidea. Entrambi i modelli di malattia possono essere inoltre utili

Proposto da Francesco Trimarchi.

Informazioni Supplementari La versione online contiene materiale supplementare disponibile su https://doi.org/10.1007/s40619-021-00833-4.

$凶$ L. Persani luca.persani@unimi.it

1 Dipartimento di Biotecnologie Mediche e Medicina Traslazionale, Università degli Studi di Milano, Milano, Italia

2 Laboratorio Sperimentale di Ricerche Endocrino-Metaboliche, IRCCS Istituto Auxologico Italiano, Milano, Italia per lo screening di nuovi farmaci e molecole che potranno essere utili per pianificare i successivi studi clinici.

Parole chiave Zebrafish - Sviluppo tiroideo - Organogenesi tiroidea $\cdot$ Genetica tireopatie

\section{Introduzione}

Gli ormoni tiroidei (OT) tiroxina (T4) e triioditironina (T3) sono essenziali per il corretto sviluppo embrionale e per la regolazione dell'omeostasi e del metabolismo nella vita post-natale. La sintesi e la secrezione degli OT da parte della tiroide è sotto il controllo dell'asse ipotalamo-ipofisi il quale, tramite meccanismi di feedback positivi e negativi, garantisce un adeguato livello di ormoni circolanti. A livello tissutale, specifici trasportatori (es. Mct8) ed enzimi ad azione desiodasica aggiungono un ulteriore livello di controllo sulla concentrazione intracellulare di OT [1, 2]. Nella maggior parte delle specie, le desiodasi di tipo I e II (Dio1 e Dio2) convertono la T4 nella forma biologicamente attiva T3, mentre la desiodasi di tipo III (Dio3) inattiva la T3 a T2 e la T4 a rT3. Una volta entrata nella cellula, la T3 si lega a specifici recettori nucleari (TRs) formando un complesso molecolare in grado di attivare o reprimere l'espressione di geni target.

L'azione degli OT dipende quindi da: 1) funzionalità dell'asse ipotalamo-ipofisi-tiroide; 2) un efficiente trasporto attraverso le membrane cellulari; 3 ) un corretto metabolismo intracellulare operato dalle desiodasi; e 4) un efficace legame della T3 ai recettori nucleari e regolazione dell'espressione genica. Mutazioni a carico di uno dei geni coinvolti in queste fasi si associa a differenti patologie tiroidee (es. ipotiroidismo congenito, difetti di trasporto degli OT, resistenza agli OT) [2-4]. Sebbene i meccanismi patogenetici 


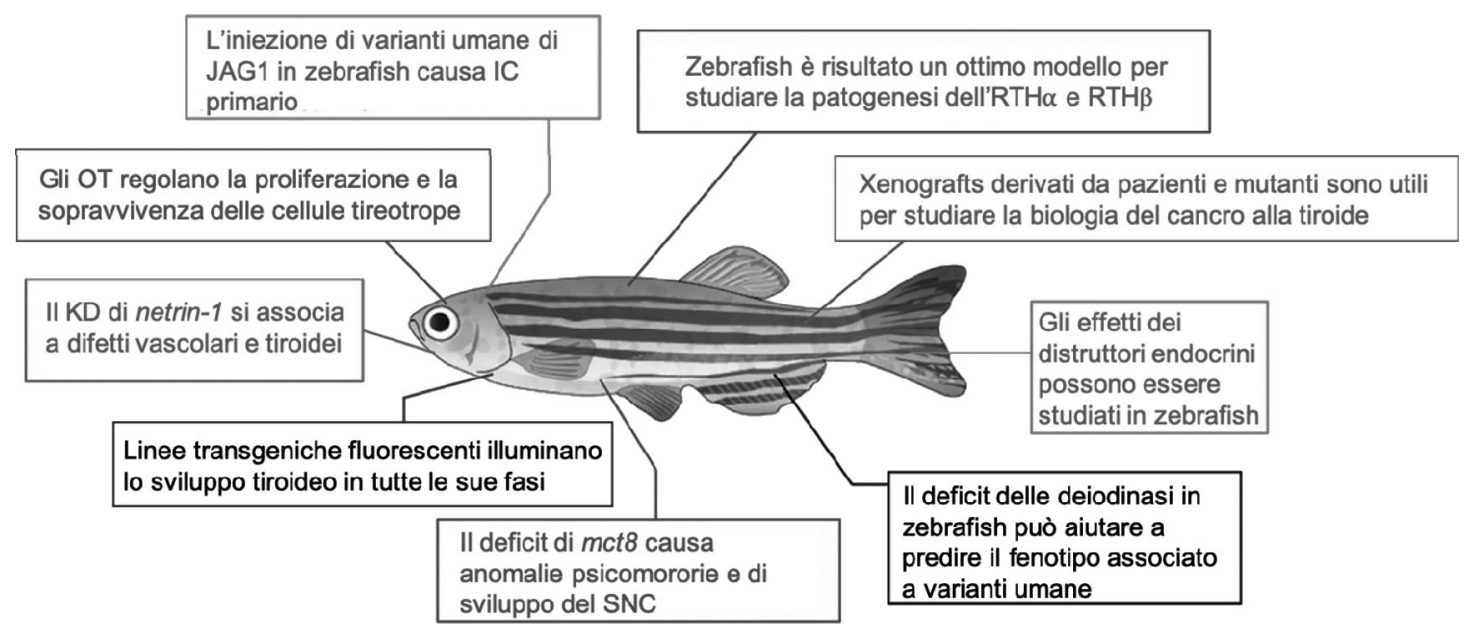

Fig. 1 Illustrazione schematica delle scoperte più recenti relative allo sviluppo della tiroide e all'azione dell'ormone tiroideo che sono state ottenute usando zebrafish come sistema modello. $O T$, ormoni tiroidei;
$I C$, ipotiroidismo congenito; $R T H$, sindrome da resistenza agli ormoni tiroidei; $S N C$, sistema nervoso centrale; $K D$, knockdown

sporadica, ma recenti studi supportano una possibile origine oligogenica dell'IC, la cui patogenesi sembra frequentemente dipendere dalla combinazione di alleli ipomorfi, di geni evolutivi o funzionali [3]. Tuttavia, i meccanismi molecolari alla base dell'IC, e in particolare della disgenesia tiroidea, rimangono in gran parte sconosciuti. Ciò è, in parte, dovuto alla nostra comprensione ancora limitata su come la funzione dei fattori intrinseci (es. fattori di trascrizione specifici della tiroide) e dei segnali di segnalazione estrinseca (es. morfogeni e fattori di crescita) si combinano tra loro durante lo sviluppo della tiroide.

Lo studio dell'anatomia tiroidea e delle sue alterazioni dopo manipolazione genetica utilizzando il modello zebrafish offre numerosi vantaggi che potrebbero superare alcuni dei limiti tecnici associati all'utilizzo di modelli di mammiferi. Contrariamente allo sviluppo intrauterino nei mammiferi, gli embrioni di zebrafish e le larve si sviluppano esternamente e sono otticamente trasparenti, rendendoli accessibili alla manipolazione sperimentale e alle osservazioni in tempo reale durante l'intero sviluppo embrionale. Queste proprietà, abbinate alla disponibilità di embrioni transgenici che esprimono proteine reporter fluorescenti in specifici tipi di cellule, consentono la visualizzazione e lo studio dello sviluppo degli organi, incluso quello della ghiandola tiroidea. Opitz e collaboratori hanno sviluppato un pannello di linee transgeniche di zebrafish in cui il promotore della tireoglobulina ( $\mathrm{Tg}$ ) è stato utilizzato per guidare l'espressione di diverse proteine fluorescenti che consentono la visualizzazione in tempo reale dei cambiamenti dinamici di dimensioni, forma e posizione della tiroide zebrafish in via di sviluppo $[5,7]$.

Inoltre, la disponibilità di strumenti per il knockdown transiente con morfolino (MO) o il knockout/knockin stabile (es. sistemi CRISPR/Cas9, Zinc Finger Nuclease [ZNF] e
Nell'uomo, l'ipotiroidismo congenito (IC) rappresenta il di-
sturbo endocrino congenito più comune, che colpisce $3-6$ su 10.000 neonati. È classicamente descritto come una malattia 


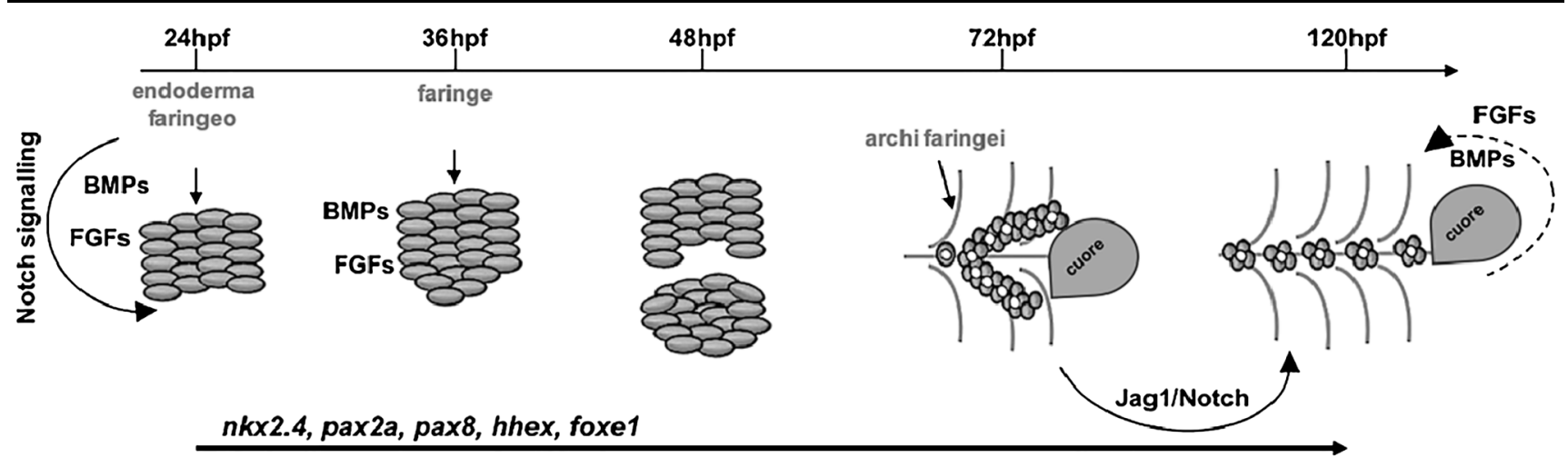

tg, jag1a

Fig. 2 Illustrazione schematica dello sviluppo della tiroide zebrafish. Il primordio tiroideo evagina dall'endoderma faringeo circa 24 ore dopo la fecondazione (hpf) ed esprime i fattori trascrizionali tiroidei $n k x 2.4$, pax $2 a$, pax 8 , hhex e foxe 1 . La differenziazione dei precursori tiroidei continua fino a $48 \mathrm{hpf}$, stadio in cui inizia l'espressione dei geni differenziativi $t g$ e slc5a5. Lo sviluppo della tiroide zebrafish è completo a

TALEN) di un gene di interesse facilita l'analisi dell'impatto di una determinata mutazione durante lo sviluppo della tiroide. La scelta di utilizzare un sistema di editing genetico rispetto a un altro, ad esempio tra MO e CRISPR/Cas9, dipende dal tipo di studio che si intende fare. I MO sono strumenti validati e ampiamente utilizzati per il knockdown funzionale e transiente di geni, mentre il sistema CRIPSR/Cas9 è un toolbox integrato che permette la perdita o il guadagno di funzione in geni target [8]. I MO sono progettati per studiare i fenotipi nelle prime fasi di sviluppo di zebrafish in quanto la sua efficacia è transitoria e tende a svanire entro 5 giorni dall'iniezione. Inoltre, è necessario tenere in considerazione la presenza di effetti off-target, che possono essere frequenti se il knockdown di un determinato gene necessita di un'alta dose di MO. I mutanti CRISPR/Cas9 sono invece stabili e permettono l'analisi dei fenotipi patologici in tutte le fasi dello sviluppo. Tuttavia, mentre il MO viene iniettato in embrioni allo stadio di 1-2 cellule (detti morfanti) e i fenotipi sono subito analizzabili negli stessi embrioni, i mutanti CRISPR/Cas9 (detti crispanti) necessitano di tempi più lunghi. La validazione della mutazione e la conferma della trasmissione nella linea germinale fa sì che si possa iniziare a studiare il fenotipo di interesse dalla generazione F2 in poi. È importante, inoltre, segnalare che è stata riportata una discrepanza nei fenotipi tra embrioni morfanti e crispanti per lo stesso gene [9]. In questo studio è stato descritto che, al contrario di embrioni morfanti che erano stati riportati mimare in modo efficiente i fenotipi osservati nell'uomo
72 hpf. Da questo momento, i follicoli tiroidei proliferano e si localizzano lungo l'aorta dorsale e iniziano a produrre T4. Fattori intrinseci ed estrinseci (Jag1-Notch, BMP e FGF) svolgono un ruolo chiave durante l'organogenesi tiroidea. Per una descrizione dettagliata dei meccanismi molecolari alla base della morfogenesi tiroidea di zebrafish, rimandiamo il lettore alla review di Fagman e Nilsson [11]

o in altri modelli sperimentali, i crispanti apparivano normali. È stato descritto che il knockout genico completo operato dal sistema CRISPR/Cas9 è associato all' assenza del fenotipo atteso a causa dell'induzione imprevedibile di una compensazione genetica da parte di altri geni correlati in grado di recuperare o mascherare il fenotipo descritto con il MO [10]. È interessante notare che questo tipo di compensazione non avviene con MO. Pertanto, entrambi gli approcci basati su MO e CRISPR/Cas9 possono essere preziosi e la loro applicazione dipende dagli obiettivi dello studio.

Nonostante le differenze anatomiche, i meccanismi molecolari dell'organogenesi tiroidea sono ben conservati tra zebrafish e mammiferi. Per una descrizione dettagliata dello sviluppo tiroideo in zebrafish e degli eventi molecolari sottostanti si consiglia la lettura della Rassegna di Fagman e Nilsson [11].

Durante i primi tre giorni di sviluppo, la ghiandola tiroidea di zebrafish non è ancora formata e gli embrioni dipendono dagli OT materni immagazzinati nel tuorlo o sacco vitellino [5, 12]. Come nei mammiferi, lo sviluppo della tiroide inizia con il differenziamento dei precursori endodermici seguita dall'evaginazione del primordio tiroideo dal pavimento faringeo. I progenitori tiroidei si differenziano successivamente in follicoli tiroidei funzionalmente attivi che migrano come unità distinte e si localizzano caudalmente all'aorta ventrale (Fig. 2). Esperimenti di knockdown in zebrafish hanno dimostrato che i fattori di trascrizione $n k x 2.1$, pax $2 a$ e hhex sono necessari per la specificazione e la diffe- 
renziazione del primordio tiroideo [11]. L'unica eccezione è foxe 1 , che regola la migrazione del primordio tiroideo nei vertebrati superiori e ricapitola il quadro dell'ectopia tiroidea in quasi il 50\% dei topi nulli per Foxe1 [12]. In zebrafish, foxel è espresso nei progenitori tiroidei, ma non sono stati osservati effetti sulla morfogenesi tiroidea negli embrioni zebrafish iniettati con MO di foxel [13]. Gli autori sostengono che la tiroide di zebrafish si sviluppa molto precocemente durante lo sviluppo e il ruolo di foxel potrebbe essere ridondante in questo contesto; è quindi possibile che il ruolo di foxel nello sviluppo della tiroide nei mammiferi sia stato acquisito durante l'evoluzione.

A 4-5 giorni post-fertilizzazione la tiroide di zebrafish è completamente sviluppata e inizia a produrre OT, i cui livelli sono finemente regolati dall'asse ipotalamo-ipofisi-tiroide. Tonyushkina e collaboratori, generando una linea transgenica che esprime GFP sotto il controllo del promotore del $t s h$ $b a$ (ortologo del TSH $\beta$ umano), hanno osservato che la regolazione del feedback negativo dell' asse ipotalamo-tiroide avviene dopo quattro giorni dalla fecondazione, stadio in cui la ghiandola tiroidea è già attiva [14]. Gli autori hanno inoltre dimostrato che un'esposizione precoce a livelli elevati o bassi di OT porta rispettivamente alla morte o iperplasia delle cellule tireotrope [15]. Questi dati dimostrano lo sviluppo tiroideo in zebrafish è TSH-indipendente e che i cambiamenti transitori di OT incidono profondamente sulla popolazione delle cellule tireotrope durante un periodo critico di sviluppo ipofisario e possono avere implicazioni a lungo termine per il set-point del TSH durante la vita adulta.

Riguardo la validazione di geni candidati durante lo sviluppo tiroideo, nei nostri studi abbiamo utilizzato il modello zebrafish per analizzare le conseguenze funzionali di diverse varianti JAG1 umane. JAG1 è un ligando dei recettori Notch le cui mutazioni inattivanti sono state identificate in pazienti con IC [16, 17]. Abbiamo osservato che il knockdown degli ortologhi di zebrafish jagla e jaglb causa ipoplasia tiroidea ed elevazione del tshba, condizioni che possono essere recuperate con l'iniezione di JAG1 wild-type umano. Confermando i risultati osservati in vitro, la co-iniezione con le varianti JAG1 umane nei morfanti di zebrafish non è in grado di recuperare, a vari gradi, i difetti associati, sottolineando l'utilità del modello zebrafish per studiare il contributo di JAG1/Notch alla funzione tiroidea.

Pappalardo e colleghi hanno usato zebrafish per studiare il ruolo di Taz, un co-regolatore trascrizionale, nella formazione della tiroide [18]. Il trascritto di wwtrl (codifica per Taz in zebrafish) è espresso precocemente nel primordio tiroideo e nel tessuto faringeo, ma il suo knockdown ha effetti limitati sull'espressione dei fattori di trascrizione e dei geni di differenziamento tiroideo nei primi stadi di sviluppo. Tuttavia, a 4-5 giorni, le larve morfanti mostrano un numero ridotto di cellule follicolari tiroidee, indicando che la proteina Taz è necessaria per la normale morfogenesi tiroidea in zebrafish [18]. Sebbene non siano state ancora identificate mutazioni in TAZ in pazienti con IC, questo lavoro suggerisce la presenza di altri geni, finora non identificati, che possono concorrere all'eziopatogenesi dell'IC.

Opitz et al. hanno recentemente descritto il ruolo di NETRIN- 1 nella patogenesi del'IC. Sono state identificate mutazioni nel gene NETRIN-1 in pazienti con disgenesia tiroidea e cardiopatia congenita [19]. In zebrafish $n t n l a$ è espresso negli archi faringei e nel mesenchima, ma non nel tessuto tiroideo. Embrioni morfanti per ntnla presentano uno sviluppo difettoso dell'aorta e una morfogenesi anomala della tiroide. Il fenotipo tiroideo è quindi il risultato della mancanza di una guida adeguata esercitata dal sistema vascolare displastico. Considerando la stretta relazione spazio-temporale tra tiroide e sviluppo dei vasi e la forte influenza dei segnali provenienti dal mesoderma cardiaco sullo sviluppo della tiroide, come BMP e FGF, netrin-1 è un ottimo candidato per avere un ruolo diretto durante lo sviluppo della tiroide e nella patogenesi dell'IC.

\section{Zebrafish e cancro della tiroide}

Zebrafish è stato recentemente utilizzato come modello animale per studiare la biologia del cancro alla tiroide. Nel nostro laboratorio è stato settato un protocollo di xenotrapianto di cellule tumorali in embrioni di zebrafish istituendo una piattaforma per lo screening di farmaci antitumorali [20]. Gli xenotrapianti derivanti da pazienti con carcinoma papillare della tiroide stimolano la migrazione dei vasi sanguigni verso l'impianto, al contrario di quanto accade con le cellule prelevate da tessuto tiroideo sano [21].

Più recentemente, è stato riportato un nuovo modello di carcinoma tiroideo in zebrafish [22]. Attraverso l'uso dell'imaging in vivo, gli autori hanno studiato una linea di zebrafish che esprime BRAFV600E nei tireociti, osservando lo sviluppo di un carcinoma invasivo nelle fasi iniziali dell'organogenesi tiroidea e che altera la struttura del follicolo in formazione. È interessante notare che il trattamento combinatorio con inibitori di BRAF e MEK è in grado di riportare allo stato precedente gli effetti sullo sviluppo indotti da BRAFV600E. Studi di espressione genica hanno inoltre identificato TWIST2 come un effettore cruciale a valle del BRAF. Gli autori hanno utilizzato il sistema CRISPR/Cas9 per inattivare geneticamente l'ortologo twist 2 , sopprimendo in questo modo gli effetti di BRAFV600E e ripristinando la morfologia della tiroide e la sintesi ormonale. Questi studi hanno dimostrato che TWIST2 è coinvolto in una fase iniziale della trasformazione mediata da BRAFV600E.

\section{Zebrafish e trasporto degli ormoni tiroidei}

Una volta prodotti e rilasciati nel sangue, gli OT necessitano di essere trasportati attraverso la membrana plasmatica 
per accedere ai compartimenti intracellulari. Il trasportatore monocarbossilato 8 (MCT8) e 10 (MCT10) e le proteine di trasporto di anioni organici (OATP) sono tutti membri della superfamiglia dei trasportatori del soluto in grado di trasportare specificatamente gli OT [22]. In particolare, MCT8 (anche chiamato SLC16A2) è necessario per il trasporto della T3 nei neuroni cerebrali, uno dei più importanti bersagli cellulari della T3 [23]. L'importanza biologica dell'MCT8 è dimostrata dalle manifestazioni della sindrome di AllanHerndon-Dudley (AHDS) causata da mutazioni disfunzionali nel gene MCT8. L'AHDS è una malattia legata all'X caratterizzata da grave ritardo psicomotorio, ipotonia e ipoplasia muscolare, atassia e compromissione della funzione cognitiva. Il meccanismo patologico sottostante è ancora da chiarire e non è nota alcuna cura per questa condizione. I topi knockout per MCT8 mostrano le stesse alterazioni nell'omeostasi degli OT dei casi di AHDS ma senza alcuna disfunzione cerebrale o disabilità locomotoria [24]. Per migliorare la comprensione dell'AHDS, il modello zebrafish è stato ampiamente utilizzato negli ultimi anni. Vatine e colleghi hanno isolato il promotore di zebrafish met8, generando una linea transgenica che conferma che $m c t 8$ si esprime principalmente nei neuroni, nelle cellule gliali e nel sistema vascolare, come nei mammiferi [25]. Il primo modello di MCT8 è stato generato un anno dopo dallo stesso gruppo usando il knockdown genico basato su MO [26]. I morfanti di mct8 mostravano difetti di locomozione e uno sviluppo difettoso del cervelletto, del contorno del mesencefalo e la presenza dei cluster apoptotici nel tessuto cerebrale. Coerentemente con quando descritto nei pazienti con AHDS, gli autori hanno osservato un'alterata espressione del $t s h b a$, dei trasportatori e delle desiodasi nei morfanti di mct8. Più recentemente, lo stesso gruppo di ricerca ha sviluppato un modello di zebrafish knock-out di MCT8 (mct8 -/-) utilizzando il sistema di editing genetico mediato dalla nucleasi di zinco (ZFN) [27]. Analogamente ai pazienti e ai morfanti, le larve met8 -/- mostravano una ridotta attività locomotoria e una carenza nelle prestazioni comportamentali, probabilmente a causa di uno sviluppo alterato dei neuroni sensoriali e motori. Inoltre, i mutanti met8 -/- hanno evidenziato anomalie neurologiche e ridotta mielinizzazione nel sistema nervoso centrale. L'analisi farmacologica ha mostrato che analoghi degli OT e clemastina possono parzialmente ridurre l'ipomielinizzazione nel sistema nervoso centrale delle larve mct8 -/-. Inoltre, il trattamento con T3 è stato in grado di impedire l'ipomielinizzazione in embrioni mct8-/- prima della maturazione della barriera emato-encefalica (BEE), ma non nelle fasi successive [28]. Nel loro insieme, questi risultati confermano l'utilità di zebrafish come modello animale per studiare la fisiopatologia della tiroide e l'AHDS, confermando l'efficacia di alcuni trattamenti farmacologici e della terapia genica mirata alla $\mathrm{BEE}$ che può migliorare la mielinizzazione nell'AHDS e possibilmente in altri disturbi cerebrali dipendenti dagli OT [29].

\section{Zebrafish e metabolismo degli ormoni tiroidei}

Differenti tipi cellulari nelle diverse fasi di sviluppo richiedono concentrazioni variabili di OT. I trasportatori di membrana regolano l'ingresso degli OT nelle cellule in modo da fornire il corretto apporto di ormoni intracellulari, il quale viene ulteriormente perfezionato dal metabolismo intracellulare controllato da tre selenoenzimi ad azione desiodasica (Dio1-3). L'unico difetto ereditario del metabolismo degli OT finora noto è quello risultante da mutazioni recessive del gene $S E C I S B P 2$ (in breve, $S B P 2$ ), la cui funzione è quella di mediare l'inserzione della selenocisteina durante la sintesi delle selenoproteine, classe eterogenea di proteine di cui fanno parte le desiodasi [29]. Lo studio dei difetti $s b p 2$ in zebrafish è attualmente in fase di pubblicazione. Non sono stati ancora segnalati casi di umani con mutazioni nei geni della desiodasi. Tuttavia, studi su modelli animali hanno anticipato che mutazioni a carico delle desiodasi potrebbero avere fenotipi variabili. Studi in zebrafish hanno dimostrato che la dio2 è necessaria per regolare la biodisponibilità di OT durante lo sviluppo embrionale. Gli embrioni knockdown per dio2, ottenuti mediante microiniezione di MO, presentano ritardo di sviluppo, mentre il knockdown di diol ha effetti di minore entità [30]. Houbrechts e collaboratori hanno generato due diverse linee di zebrafish mutanti per la dio2 tramite il sistema delle ZFN. Entrambi i mutanti presentano una completa inattività della dio $2 \mathrm{e}$ una conseguente riduzione della concentrazione di T3 nei tessuti [31]. I mutanti mostrano inoltre difetti dello sviluppo precoci, alterata attività locomotoria ed effetti a lungo termine sulla crescita e sulla fertilità. L'azione della dio3 (codificata in zebrafish da due geni, dio3a e dio3b) è stata inizialmente studiata in relazione alla rigenerazione delle pinne. Heijlen e colleghi hanno recentemente dimostrato che il knockdown mediato dai MO di dio3a o dio3b si traduce in una parziale predita di attività della dio3, indicando che entrambi i geni codificano per enzimi attivi [30]. Inoltre, il doppio knockdown di dio3a/dio3b causa ritardo di sviluppo embrionale, ridotta attività locomotoria e difetti di sviluppo retinico [32, 33]. Lo stesso gruppo di ricerca ha successivamente analizzato le variazioni del trascrittoma dovute al doppio knockdown diol/dio2 e dio3aldio3b in larve di zebrafish. Entrambe le condizioni hanno comportato un'espressione differenziale dei geni coinvolti nel metabolismo energetico e nello sviluppo muscolare [34]. L'up-regolazione dei trascritti che codificano per le proteine ATP-dipendenti sembra riflettere una risposta compensativa a un ridotto tasso metabolico, una condizione legata all'ipotiroidismo osservato nei doppi knockdown diol/dio2. I doppi morfanti dio3aldio3b presentano invece una up-regolazione dei geni coinvolti nell'incremento del tasso metabolico, nella stimolazione della gluconeogenesi e della frequenza cardiaca. Questi risultati 
forniscono nuove informazioni sul ruolo delle desiodasi durante lo sviluppo, evidenziando l'importanza di un corretto equilibrio di OT durante lo sviluppo dei vertebrati.

\section{Zebrafish e azione degli ormoni tiroidei}

Nell'uomo, l'azione degli OT è mediata da diversi recettori nucleari (TR $\alpha 1$ e $\operatorname{TR} \alpha 2, \operatorname{TR} \beta 1$ e $\operatorname{TR} \beta 2$ ) codificati da due geni (THRA e THRB), con diversa distribuzione tissutale. Fino a qualche anno fa, il termine RTH veniva applicato al fenotipo associato a mutazioni dominanti-negative (DN) nel gene THRB. Con l'identificazione delle mutazioni DN nel gene THRA, che presenta un fenotipo estremamente diverso, le sindromi sono ora identificate come $\mathrm{RTH} \beta$ e RTH $\alpha$ [35]. Le caratteristiche di base dell'RTH $\beta$ comprendono alti livelli di OT circolanti, TSH non soppresso e gozzo insieme a manifestazioni cliniche variabili dovute all'ipotiroidismo nei tessuti che esprimono principalmente il TR $\beta$ e agli effetti tireotossici nei tessuti che maggiormente esprimono il $\mathrm{TR} \alpha$ (es. ansia, tachicardia, disturbi dell'udito e della visione dei colori, disturbo da deficit di attenzione e iperattività, bassa densità ossea). In zebrafish il thrb (che codifica che le isoforme TR $\beta 1$ e TR $\beta 2$ ) è espresso principalmente nella retina, nell'ipofisi e nella vescicola otica durante le prime fasi di sviluppo embrionale [36]. La generazione di un modello di DN mediante microiniezione MO ha dimostrato che le isoforme $\operatorname{TR} \beta$ sono necessarie per il corretto sviluppo embrionale, in particolare degli organi sensoriali (occhi e vescicola otica). Inoltre, i morfanti del TR $\beta$ hanno mostrato la tipica firma biochimica dell'RTH $\beta$ (alti livelli di OT, tshba elevato e ridotta sensibilità dell'ipofisi al trattamento con L-T4) [36]. Suzuki e colleghi hanno generato una linea transgenica fluorescente per il TR $\beta 2$ dimostrando che la differenziazione di coni di tipo L (lunghi) è dipendente dall'attività del TR $\beta 2$ [37]. Il knockdown di TR $\beta 2$ causa un ridotto differenziamento dei coni $\mathrm{L}$ e un concomitante aumento dei coni UV. Al contrario, l'espressione ectopica di TR $\beta 2$ dopo la differenziazione dei coni produce coni con espressione di opsina mista.

Dal 2012, diverse mutazioni eterozigoti nel gene THRA sono state descritte in pazienti con RTH $\alpha$ 4. I sintomi associati si sovrappongono a quelli dei pazienti con IC non trattato (ritardo della crescita, disturbi psico-neuromotori, sviluppo osseo ritardato e bradicardia) ma con un elevato rapporto T3/T4 e livelli normali o leggermente aumentati di TSH. Tutte le mutazioni si localizzano nel dominio deputato al legame alla T3 (ligand binding domain, LBD) e mostrano, con diversi gradi di gravità, una ridotta affinità all'ormone $\mathrm{o}$ una difettosa interazione con i co-repressori o co-attivatori. Di conseguenza, i pazienti con RTH $\alpha$ presentano risposte variabili al trattamento con OT [4]. Durante lo sviluppo embrionale di zebrafish, il thraa (gene che codifica per le iso- forme $\mathrm{TR} \alpha 1$ e TR $\alpha 2$ ) è principalmente espresso nel sistema nervoso centrale, nel cuore, nel tratto gastrointestinale e nei dotti pronefrici. Gli embrioni recanti mutazioni DN nell'LBD delle isoforme TR $\alpha$ di zebrafish presentano una serie di fenotipi paragonabili a quelli riportati nei pazienti con $\mathrm{RTH} \alpha$ (es. difetti di crescita, alterato sviluppo delle cartilagini, anemia e alto rapporto T3/T4) [36]. I morfanti thraa presentano una ridotta espressione di dio3a e dio3b e un'aumentata espressione della dio2 a livello ipotalamico, condizioni che possono spiegare l'alterazione dei livelli di OT osservati.

L'iniezione di trascritti wild-type e mutati del TR $\alpha$ umano ha dimostrato che i recettori di zebrafish e uomo sono funzionalmente intercambiabili. L'iniezione dei trascritti mutanti causa profonde alterazioni morfologiche, neurologiche, cardiovascolari, ematologiche, scheletriche e biochimiche che mimano le manifestazioni dell'RTH $\alpha$. Coerentemente con i risultati ottenuti in vitro, il trattamento con T3 ad alte dosi è in grado di normalizzare i fenotipi negli embrioni iniettati con le varianti missenso del $\mathrm{TR} \alpha$ (ridotta affinità alla T3), mentre le mutazioni tronche (difettosa interazione con i cofattori) sono completamente refrattarie al trattamento [38].

Questi dati dimostrano che una corretta azione degli ormoni tiroidei è necessaria per lo sviluppo embrionale di zebrafish e che l'elevata omologia genetica rende il modello estremamente utile per studiare gli effetti delle varianti umane.

\section{Zebrafish e distruttori endocrini}

Negli ultimi anni sta crescendo l'utilizzo di zebrafish per analizzare gli effetti delle sostanze chimiche che alterano il sistema endocrino (EDC). Alcuni studi hanno valutato gli effetti dell'esposizione a bisfenolo AF o S (BPAF o BPS) [39-41]. Il trattamento con questi inquinanti induce alterazioni variabili nelle concentrazioni di OT, nonché nel metabolismo e nell'azione di OT. Questi dati forniscono interessanti spunti sulla tossicità di questi composti ma sono spesso di difficile interpretazione in quanto sono stati osservati fenotipi opposti (diminuzione o aumento delle concentrazioni di tiroxina) e risultati contrastanti riguardo alle variazioni dell'espressione genica in sia i tessuti tiroidei che ipotalamo-ipofisari dopo trattamento con BPAF o BPS [39-41].

\section{Conclusioni}

In questa Rassegna abbiamo sintetizzato i risultati relativi allo sviluppo della tiroide e all'azione degli OT che sono stati ottenuti negli zebrafish negli ultimi anni (Fig. 1). Tutti 
questi dati evidenziano il grande potenziale di questo modello per studiare le malattie della tiroide. Gli embrioni di zebrafish sono particolarmente utili grazie alle loro dimensioni ridotte, al rapido sviluppo e alla trasparenza ottica. Data la conservazione dei meccanismi molecolari che regolano lo sviluppo della tiroide e l'azione degli OT tra zebrafish e mammiferi, la disponibilità di reporter fluorescenti e diverse tecniche di manipolazione genetica rendono zebrafish un potente modello per studiare i meccanismi alla base delle malattie tiroidee. La generazione di specifici modelli genetici può sfruttare efficacemente i vantaggi degli embrioni di zebrafish per una caratterizzazione fenotipica di nuovi geni candidati. Inoltre, questi modelli possono essere sfruttati per lo screening dei farmaci permettendo quindi di aiutare e accelerare lo sviluppo di nuovi protocolli di trattamento.

Funding Note Open access funding provided by Università degli Studi di Milano within the CRUI-CARE Agreement.

Conflitto di interesse Gli autori Federica Marelli e Luca Persani dichiarano di non avere conflitti di interesse.

Consenso informato Lo studio presentato in questo articolo non ha richiesto sperimentazione umana.

Studi sugli animali In questo articolo gli autori hanno riassunto i risultati di esperimenti su zebrafish eseguiti secondo le norme vigenti in Italia e all'estero e approvati dai competenti organi istituzionali.

Nota della casa editrice Springer Nature rimane neutrale in riguardo alle rivendicazioni giurisdizionali nelle mappe pubblicate e nelle affiliazioni istituzionali.

Open Access This article is licensed under a Creative Commons Attribution 4.0 International License, which permits use, sharing, adaptation, distribution and reproduction in any medium or format, as long as you give appropriate credit to the original author(s) and the source, provide a link to the Creative Commons licence, and indicate if changes were made. The images or other third party material in this article are included in the article's Creative Commons licence, unless indicated otherwise in a credit line to the material. If material is not included in the article's Creative Commons licence and your intended use is not permitted by statutory regulation or exceeds the permitted use, you will need to obtain permission directly from the copyright holder. To view a copy of this licence, visit http://creativecommons.org/licenses/by/4.0/.

\section{Bibliografia}

1. Medici M, Visser TJ, Peeters RP (2017) Genetics of thyroid function. Best Pract Res Clin Endocrinol Metab 31(2):129-142

2. Dumitrescu AM, Refetoff S (2013) The syndromes of reduced sensitivity to thyroid hormone. Biochim Biophys Acta 1830(7):3987-4003

3. de Filippis T, Gelmini G, Paraboschi E et al (2017) A frequent oligogenic involvement in congenital hypothyroidism. Hum Mol Genet 26(13):2507-2514

4. Moran C, Chatterjee K (2015) Resistance to thyroid hormone due to defective thyroid receptor alpha. Best Pract Res Clin Endocrinol Metab 29(4):647-657
5. Opitz R, Antonica F, Costagliola S (2013) New model systems to illuminate thyroid organogenesis. Part I: an update on the zebrafish toolbox. Eur Thyroid J 2(4):229-242

6. Porazzi P, Calebiro D, Benato F et al (2009) Thyroid gland development and function in the zebrafish model. Mol Cell Endocrinol 312(1-2):14-23

7. Opitz R, Maquet E, Huisken J et al (2012) Transgenic zebrafish illuminate the dynamics of thyroid morphogenesis and its relationship to cardiovascular development. Dev Biol 372(2):203-216

8. Varshney GK, Sood R, Burgess SM (2015) Understanding and editing the zebrafish genome. Adv Genet 92:1-52

9. Kok FO, Shin M, Ni CW et al (2015) Reverse genetic screening reveals poor correlation between morpholino-induced and mutant phenotypes in zebrafish. Dev Cell 32(1):97-108

10. Rossi A, Kontarakis Z, Gerri C et al (2015) Genetic compensation induced by deleterious mutations but not gene knockdowns. Nature 524(7564):230-233

11. Fagman H, Nilsson M (2011) Morphogenetics of early thyroid development. J Mol Endocrinol 46(1):R33-42

12. De Felice M, Ovitt C, Biffali E et al (1998) A mouse model for hereditary thyroid dysgenesis and cleft palate. Nat Genet 19:399401

13. Nakada C, Iida A, Tabata Y et al (2009) Forkhead transcription factor foxe1 regulates chondrogenesis in zebrafish. J Exp Zool B Mol Dev Evol 312(8):827-840

14. Tonyushkina KN, Shen MC, Ortiz-Toro T et al (2014) Embryonic exposure to excess thyroid hormone causes thyrotrope cell death. J Clin Invest 124(1):321-327

15. Tonyushkina KN, Krug S, Ortiz-Toro T et al (2017) Low thyroid hormone levels disrupt thyrotrope development. Endocrinology 158(9):2774-2782

16. de Filippis T, Marelli F, Nebbia G et al (2016) JAG1 loss-offunction variations as a novel predisposing event in the pathogenesis of congenital thyroid defects. J Clin Endocrinol Metab 101(3):861-870

17. Marelli F, Persani L (2017) Role of Jagged1-Notch pathway in thyroid development. J Endocrinol Invest, 1-7

18. Pappalardo A, Porreca I, Caputi L et al (2015) Thyroid development in zebrafish lacking Taz. Mech Dev 138(3):268-278

19. Opitz R, Hitz M, Vandernoot I et al (2015) Functional zebrafish studies based on human genotyping point to netrin-1 as a link between aberrant cardiovascular development and thyroid dysgenesis. Endocrinology 156(1):377-388

20. Gaudenzi G, Albertelli M, Dicitore A et al (2017) Patient-derived xenograft in zebrafish embryos: a new platform for translational research in neuroendocrine tumors. Endocrine 57(2):214-219

21. Cirello V, Gaudenzi G, Grassi ES et al (2017) Tumor and normal thyroid spheroids: from tissues to zebrafish. Minerva Endocrinol 43(1): $1-10$

22. Anelli V, Villefranc JA, Chhangawala S et al (2017) Oncogenic BRAF disrupts thyroid morphogenesis and function via twist expression. eLife 6:e20728

23. Groeneweg S, Visser WE, Visser TJ (2017) Disorder of thyroid hormone transport into the tissues. Best Pract Res Clin Endocrinol Metab 31(2):241-253

24. Mayerl S, Müller J, Bauer R et al (2014) Transporters MCT8 and OATP1C1 maintain murine brain thyroid hormone homeostasis. J Clin Invest 124(5):1987-1999

25. Vatine GD, Zada D, Lerer-Goldshtein T et al (2013) Zebrafish as a model for monocarboxyl transporter 8-deficiency. J Biol Chem 288(1):169-180

26. de Vrieze E, van de Wiel SM, Zethof J et al (2014) Knockdown of monocarboxylate transporter 8 (mct8) disturbs brain development and locomotion in zebrafish. Endocrinology 155(6):2320-2330 
27. Zada D, Tovin A, Lerer-Goldshtein T et al (2014) Altered behavioral performance and live imaging of circuit-specific neural deficiencies in a zebrafish model for psychomotor retardation. PLoS Genet 10(9):e1004615

28. Zada D, Tovin A, Lerer-Goldshtein T et al (2016) Pharmacological treatment and BBB-targeted genetic therapy for MCT8-dependent hypomyelination in zebrafish. Dis Model Mech 9(11):1339-1348

29. Zada D, Blitz E, Appelbaum L (2017) Zebrafish - an emerging model to explore thyroid hormone transporters and psychomotor retardation. Mol Cell Endocrinol 459:53-58

30. Heijlen M, Houbrechts AM, Darras VM (2013) Zebrafish as a model to study peripheral thyroid hormone metabolism in vertebrate development. Gen Comp Endocrinol 188:289-296

31. Houbrechts AM, Delarue J, Gabriëls IJ et al (2016) Permanent deiodinase type 2 deficiency strongly perturbs zebrafish development, growth, and fertility. Endocrinology 157(9):3668-3681

32. Heijlen M, Houbrechts AM, Bagci E et al (2014) Knockdown of type 3 iodothyronine deiodinase severely perturbs both embryonic and early larval development in zebrafish. Endocrinology 155(4):1547-1559

33. Bagci E, Heijlen M, Vergauwen L et al (2015) Deiodinase knockdown during early zebrafish development affects growth, development, energy metabolism, motility and phototransduction. PLoS ONE 10(4):e0123285

34. Houbrechts AM, Vergauwen L, Bagci E et al (2016) Deiodinase knockdown affects zebrafish eye development at the level of gene expression, morphology and function. Mol Cell Endocrinol 424:81-93
35. Refetoff S, Bassett JH, Beck-Peccoz P et al (2014) Classification and proposed nomenclature for inherited defects of thyroid hormone action, cell transport, and metabolism. Eur Thyroid J 3(1):7-9

36. Marelli F, Carra S, Agostini M et al (2016) Patterns of thyroid hormone receptor expression in zebrafish and generation of a novel model of resistance to thyroid hormone action. Mol Cell Endocrinol 424:102-117

37. Suzuki SC, Bleckert A, Williams PR et al (2013) Cone photoreceptor types in zebrafish are generated by symmetric terminal divisions of dedicated precursors. Proc Natl Acad Sci USA 110(37):15109-15114

38. Marelli F, Carra S, Rurale G et al (2017) In vivo functional consequences of human THRA variants expressed in the zebrafish. Thyroid 27(2):279-291

39. Kwon B, Kho Y, Kim PG et al (2016) Thyroid endocrine disruption in male zebrafish following exposure to binary mixture of bisphenol AF and sulfamethoxazole. Environ Toxicol Pharmacol 48:168-174

40. Tang T, Yang Y, Chen Y et al (2015) Thyroid disruption in zebrafish larvae by short-term exposure to bisphenol AF. Int J Environ Res Public Health 12(10):13069-13084

41. Zhang DH, Zhou EX, Yang ZL (2017) Waterborne exposure to BPS causes thyroid endocrine disruption in zebrafish larvae. PLoS ONE 12(5):e0176927 